\title{
Correction of blink artifacts using independent component analysis and empirical mode decomposition
}

\author{
JOB P. LINDSEN ${ }^{\mathrm{a}}$ AND JOYDEEP BHATTACHARYA ${ }^{\mathrm{a}, \mathrm{b}}$ \\ ${ }^{a}$ Department of Psychology, Goldsmiths, University of London, New Cross, London, UK \\ ${ }^{\mathrm{b}}$ Commission for Scientific Visualization, Austrian Academy of Sciences, Vienna, Austria
}

\begin{abstract}
Blink-related ocular activity is a major source of artifacts in electroencephalogram (EEG) data. Independent component analysis (ICA) is a well-known technique for the correction of such ocular artifacts, but one of the limitations of ICA is that the ICs selected for removal contain not only ocular activity but also some EEG activity. Straightforward removal of these ICs might, therefore, lead to a loss of EEG data. In this article a method is proposed to separate blinkrelated ocular activity from actual EEG by combining ICA with a novel technique, empirical mode decomposition. This combination of two techniques allows for maximizing the retention of EEG data and the selective removal of the eyeblink artifact. The performance of the proposed method is demonstrated with simulated and real data.
\end{abstract}

Eyeblinks constitute a major source of artifacts in electroencephalographic (EEG) recordings. This is primarily caused by the movement of the eyelids over the eyeball despite the lack of any concomitant movement of the eyeball (Matsuo, Peters, \& Reilly, 1975). It is suggested that the eyeball forms an electric dipole and the eyelid acts as a sliding potential source; therefore the movement of eyelids over eyeballs causes potential shifts and contaminates EEG signals (Berg \& Scherg, 1991). In general, the eyeblink artifacts are characterized by a larger amplitude and a lower frequency than the true EEG, with a fronto-polar distribution of activity that gradually declines toward posterior electrode positions; indeed, blink-related artifact amplitude is inversely proportional to the square of the distance (Croft \& Barry, 2000). A common way to remove these artifacts is to reject blink-contaminated epochs. However, a disproportionate number of trials might be discarded this way; not only are task-relevant neural responses thrown away but the power of statistical tests might also be severely reduced. A practical alternative to the rejection of artifact-laden epochs is the procedure of artifact correction, whereby the epochs are "cleaned" by eliminating the blink artifacts; various methods have been designed and compared to this end (see, for details, Croft \& Barry, 2000; Croft, Chandler, Barry, Cooper, \& Clarke, 2005; Hoffmann \& Falkenstein, 2008; Ille, Berg, \& Scherg, 2002; Joyce, Gorodnitsky, \& Kutas, 2004; Picton et al., 2000). One popular class of ocular artifact correction methods is based on regression in the time domain, that is, a proportion of the electro-oculographic (EOG) recording is "subtracted" from each scalp electrode (Gratton, Coles, \& Donchin, 1983; Quilter, MacGillivray, \& Wadbrook,

The research is supported by JST.ERATO project (J.B.).

Address reprint requests to: Joydeep Bhattacharya, Department of Psychology, Goldsmiths, University of London, New Cross, London, SE146NW, UK. E-mail: j.bhattacharya@gold.ac.uk
1977; Schlogl et al., 2007). Another popular method for ocular artifact correction is based on blind source separation, usually by means of independent component analysis (ICA), which is based on the assumption that the blink artifact is represented by a limited number of independent components (ICs). Once identified, these ICs can be selectively removed during the inverse ICA transformation to yield the clean data (Jung et al., 2000; Vigario, 1997).

A potential problem using these methods, albeit one often overlooked, is that both the recorded EOG signals and the ICs selected for removal will contain some EEG data in addition to the blink artifacts (Castellanos \& Makarov, 2006; Frank \& Frishkoff, 2007). So, using this method to correct blink artifacts is likely to result in the loss of some true EEG data as well (Barbati, Porcaro, Zappasodi, Rossini, \& Tecchio, 2004; Ille et al., 2002; Joyce et al., 2004).

In this article, a novel method is proposed for the correction of blink artifacts that aims to separate these artifacts from the EEG data, resulting in a more exclusive removal of the artifact and therefore ensuring a better reconstruction of EEG data from the artifact-contaminated epochs. Essentially, this method consists of three steps: (1) transforming the original data into ICs, (2) performing an empirical mode decomposition (EMD; Huang et al., 1998) of the ICs containing blink artifacts, and (3) classifying the intrinsic mode functions (IMFs) obtained by means of EMD as either blink or EEG and selectively removing the blink-related intrinsic modes. These three steps can be performed automatically. In the following sections the proposed method is presented and its performance in removing blink artifacts is demonstrated using simulated and real data.

\section{Selectively Removing Blink Artifacts Using ICA and EMD}

The first step in the proposed method is to transform the data by means of ICA. The underlying assumption of the ICA is that the 
signals recorded at each electrode position are linear mixtures of a number of sources with an independent time course. The aim of ICA is to find a set of spatial filters that inverts this mixture and recover the original sources, called independent components (ICs). So, if the multichannel EEG recording is considered as a mixture of brain and artifactual blink signals, ICA provides a spatial filter that captures these blink artifacts in a limited number of ICs (Jung et al., 2000). Once these ICs are identified, they can be excluded from the inverse transformation back to the signal space. The use of ICA to capture eyeblink activity is a well-established method, and discussions of its strengths and limitations can be found elsewhere (Frank \& Frishkoff, 2007; Hoffmann \& Falkenstein, 2008).

One limitation of using ICA for ocular artifact correction is that a straightforward removal of the ICs containing artifacts most likely results in some loss of EEG data, because those ICs will rarely consist of only blink-related EOG activity. Furthermore, when eyeblink activity is not captured by a single IC but spreads out over multiple components, that is, when the ICA suffers from a split variance, the amount of lost EEG data could be considerable when these multiple ICs are removed (Frank \& Frishkoff, 2007).

To retain the true EEG activity in those selected ICs containing blinks, a recently introduced technique, empirical mode decomposition, for an adaptive representation of nonstationary signals is applied (Huang et al., 1998). Unlike other signal decomposition methods, such as the Fourier or the wavelet transforms, EMD is purely data driven, that is, the basis of the decomposition is adaptively derived from the data. Furthermore, because the EMD is based on the local characteristic time scale of the data, it is applicable to nonlinear and nonstationary signals, such as large-scale brain responses (Liang, Bressler, Buffalo, Desimone, \& Fries, 2005).

The EMD procedure considers a signal or time series to be composed of one oscillation riding on top of another, slower oscillation that is, in turn, riding on still slower oscillations. Therefore, application of the EMD to a signal amounts to an iterative procedure that identifies each of these intrinsic oscillatory modes by their characteristic time scales. Here, the effective steps of EMD are briefly summarized:

1. Given a signal $x(t)$, identify all local extremes (maxima and minima)

2. Interpolate between maxima to estimate the upper envelope $x_{\text {up }}(t)$ and between minima to estimate the lower envelope $x_{\text {low }}(t)$.

3. Compute the mean of the two envelopes, $m(t)=\left(x_{\mathrm{up}}(t)+\right.$ $\left.x_{\text {low }}(t)\right) / 2$, and subtract it from the data: $d(t)=x(t)-m(t)$.

Steps 1-3 are repeated on $d(t)$ until the resulting signal, $c_{1}(t)$, satisfies the three following criteria: (1) the number of extremes and the number of zero crossings must either be equal or differ at most by one, (2) the mean value of the envelope defined by the local maxima and the envelope defined by the local minima must be zero at any time point, and (3) the difference between two consecutive repetitions, in terms of standard deviation, must not be smaller than some predetermined criterion. Once this is achieved, the resulting signal is considered to be the first IMF. After an IMF is identified it is subtracted from the original data, and the residue is taken as the new data from which the next IMF can be identified by repeating the steps that yielded the first IMF. No more IMFs are extracted from the data when the residue becomes a monotonic function or when it becomes negligibly small. Therefore, the end result of the EMD method is a set of IMFs ordered according to their characteristic time scales plus a residual that, when linearly summed together, constitute the original signal.

Although EMD ensures a complete reconstruction of the data by summing all IMFs, removing the artifactual IMFs selectively from the summation could effectively filter out the artifact from the original data. So, instead of removing the entire IC from the inverse ICA transformation, the artifact-free part of the IC can be incorporated together with other ICs to reconstruct the data. Consequently, the proposed method of combining ICA and EMD allows for the selective removal of only those modes of the IC predominantly associated with blink artifacts and minimizes the loss of true EEG data.

A crucial step in the proposed EMD-based method for the successful correction of the blink artifacts is the classification of the IMFs as either a blink- or an EEG- related mode. To fully automate the whole correction procedure, a simple criterion, albeit ad hoc, is applied for the classification of the IMFs based on their standard deviations ( $S D \mathrm{~s})$. Though the $S D$ s of the IMFs representing the EEG-related components will differ to a certain extent depending on the strengths of constituent frequency bands (i.e., alpha, beta, gamma), they will still be considerably lower than the $S D$ s of the IMFs representing the blink artifacts, because of the characteristic differences in amplitudes of EEG and blink signals. Based on this property, the following classification rule is suggested: If the $S D$ of an IMF is larger than $p$ times the $S D$ of the first IMF, then that IMF and all other lower order IMFs will be classified as blink-related modes. Simulation results that are described later in this article suggest that this classification procedure produces optimal results with $p$ between 1.5 and 2.5 .

\section{Illustration of the EMD Method}

An illustrative example of how the EMD of an IC results in multiple IMFs, which subsequently can be used to separate a superimposed blink and EEG signal, is shown in Figure 1. The top panel shows a signal (in black) that is composed of a simulated eyeblink superimposed onto an IC obtained from an ICA decomposition of 64-channel EEG data. Application of the EMD method to this signal produces seven IMFs (bottom panel). Using a $p$ ( = the $S D$ of second or higher order IMFs/the $S D$ of the first IMF) of 2, the first four IMFs (in blue) are classified as true EEG activity, that is, the $S D$ s of the second, third, and fourth IMF are smaller than 2 times the $S D$ of the first IMF. Visual inspection of these four modes confirms that they are a reasonable represention of the relatively high frequency oscillatory components in the original data and do not evidently show any characteristics of artifactual blink-related activity. The fifth IMF (in red) has a $S D$ larger than 2 times the $S D$ of the first IMF, and hence this IMF and the two remaining lower-order IMFs (in red) are classified as blink-related activity. Here, visual inspection of the IMFs confirms that these last three modes (in red) are characterized by relatively large amplitudes and low frequencies that are also observed in the blink activity. As can be seen in the top panel of Figure 1, the sum of the first four IMFs (in blue) provides a good approximation of the original IC activity (in black), whereas the sum of the last three IMFs (in red) provides a good approximation of the eyeblink activity. Therefore, this ex- 


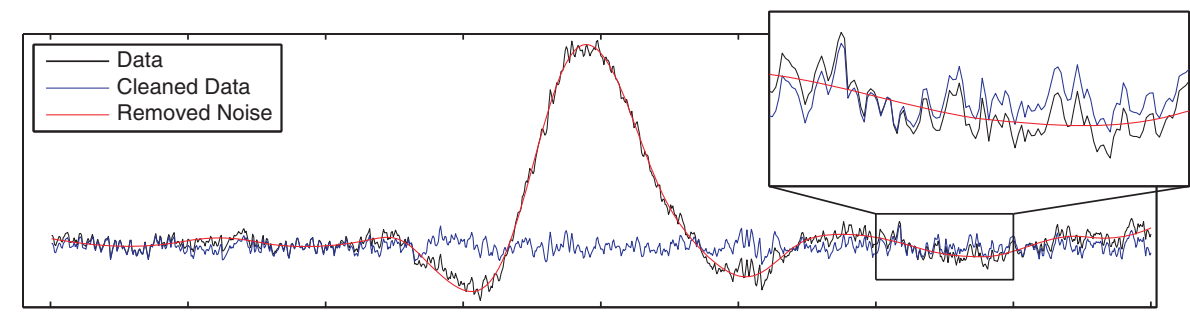

\section{Emperical Mode Decomposition}

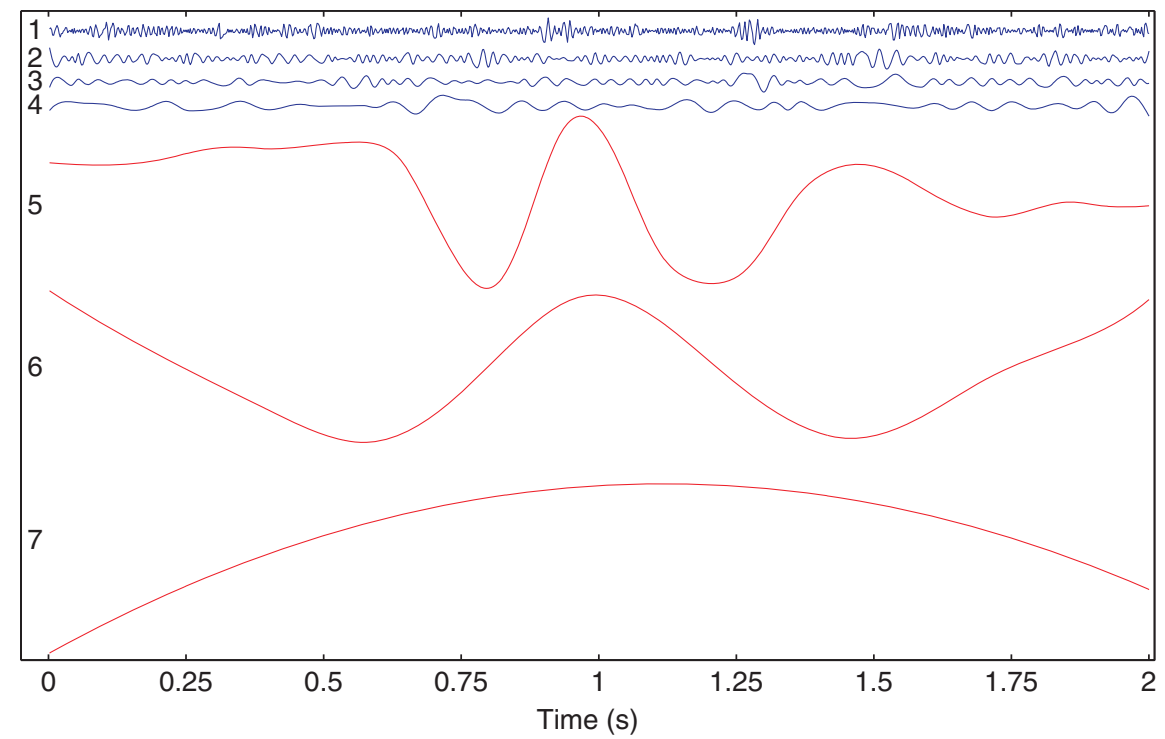

Figure 1. Empirical mode decomposition (EMD) of an EEG signal with an eyeblink artifact. The top panel shows the signal plus artifact (black line), the cleaned signal (blue line), and the artifact removed by EMD (red line). The bottom panel shows the seven independent mode functions (IMFs) generated by an EMD of the signal plus artifact. The first four IMFs (blue lines) are classified as containing EEG, and their sum is taken as the cleaned signal. The last three IMFs (red lines) are classified as containing the blink artifact, and these are removed from the signal. The inset shows in detail the differences between the original and cleaned signals.

ample shows how EMD could separate blink-related activity from neural activity based on the differences in both time scale and amplitude of intrinsic oscillatory modes.

\section{Validation of the EMD Based Method}

A major problem in EOG correction, including the eyeblink removal research, is the lack of an obvious quantitative method of validation. This is partly because, in an experimental situation, there is no access to the uncontaminated EEG activity, and therefore there is no quantitative criterion against which the performance of a correction method could be rigorously measured (Croft \& Barry, 2000). Often the success of a correction method is measured qualitatively, whereby the corrected waveforms are supposed to have face validity based on visual inspection, therefore promoting subjectivity (Verleger, Gasser, \& Mocks, 1982). In the earlier example (Figure 1), visual inspection of the IMFs that are retained in the data set do not show any blink activity, yet the IMFs that are selected for removal contains some low frequency EEG components as well. Therefore, it is important to use some additional quantitative measures in addition by which the success and the error of the correction method can be quantified, and this is only possible through simulation studies. Here a simulation was performed in which a blink template was superimposed onto artifact-free IC data and subsequently removed using EMD, allowing for a direct comparison between the original and the corrected signal. This simulation also allowed for an evaluation of the performance of the classification method based on the relative $S D$ s of the IMFs by systematically varying the values of $p$ and the size of the artifacts relative to the variation in the data.

In the simulation, 100 segments of $4 \mathrm{~s}$ (2,048 sample points) of artifact-free IC data were selected from a 64-channel EEG study conducted in our laboratory (see, for details, Lindsen, Jones, Shimojo, \& Bhattacharya, 2010). An eyeblink template of $2 \mathrm{~s}$ duration was obtained by averaging 140 peak-aligned blink intervals from 2 randomly chosen participants in this study. This eyeblink template was superimposed onto the IC data with a random offset varying between 0 and $2 \mathrm{~s}$, creating waveforms resembling those data segments containing real blink artifacts. The relative size of this artifact, defined as the peak-to-peak amplitude of the blink template, was taken as $s$ times the $S D$ of the data segment on which it was superimposed, with $s$ varying between 20 and 40 in steps of 2 . The value of $p$ (the multiplication factor that determines the upper limit of acceptable $S D$ s in the classification of IMFs) varied between 1 and 7 in steps of 0.25 .

At every pair $\{p, s\}$, EMDs were obtained for the $100 \mathrm{seg}$ ments with superimposed blink artifacts, and for each of these EMDs, the IMFs that were classified by our proposed classification method as containing blink-related components were discarded. Then, for each segment, the correlation coeffi- 
cient between the cleaned data, that is, the sum of the IMFs classified as containing EEG and the original data without the artifact, was computed as a measure of the goodness of fit. As a result, 100 values were obtained for every $\{p, s\}$ pair, and the average was taken as an indication of how much of the original signal was maintained after artifact correction. Figure 2 shows the results of this simulation. In addition to the correlation coefficient, the mutual information between the original and the corrected signal was also calculated. The mutual information is sensitive to both linear and higher order correlations between two signals whereas correlation coefficient is sensitive to only linear correlation (Li, 1990).

Over the intervals of varying artifact sizes tested in this simulation, the optimal performance of the EMD+ICA method is fairly constant between correlation values of .55 and .60 . The mutual information estimates showed a similar pattern as the correlation coefficients but were, in general, slightly lower with maximum values ranging from .35 to .4 nats. The simulation also shows that classification performance deteriorates for both too high and too low values of $p$ due to the exclusion of IMFs containing EEG data and to the inclusion of IMFs containing artifacts, respectively. The classification is optimal when $p$ is between 1.5 and 2.5.

Next, the improvement of the combined ICA and EMD method over the traditional ICA method was assessed by directly comparing the outcomes of the two methods for the data of one randomly chosen participant from the same data set used in the simulation study. An ICA decomposition of the data (64 EEG, two VEOG, and two HEOG channels) was obtained with the infomax algorithm implemented in the EEGLAB toolbox (Delorme \& Makeig, 2004). One single IC was identified by visual inspection as containing blink artifacts. First, the IC containing blink artifacts was removed before the inverse transformation recalculated the data. Next, the IMFs of the IC containing blinks were obtained with an EMD, and the IMFs representing the artifact were excluded before the inverse ICA transformation.

The difference in the amount of data lost between removing entire ICs and removing only the blink components of the ICs by means of EMD during inverse ICA transformation is illustrated qualitatively in Figure 3. This figure shows the data from five

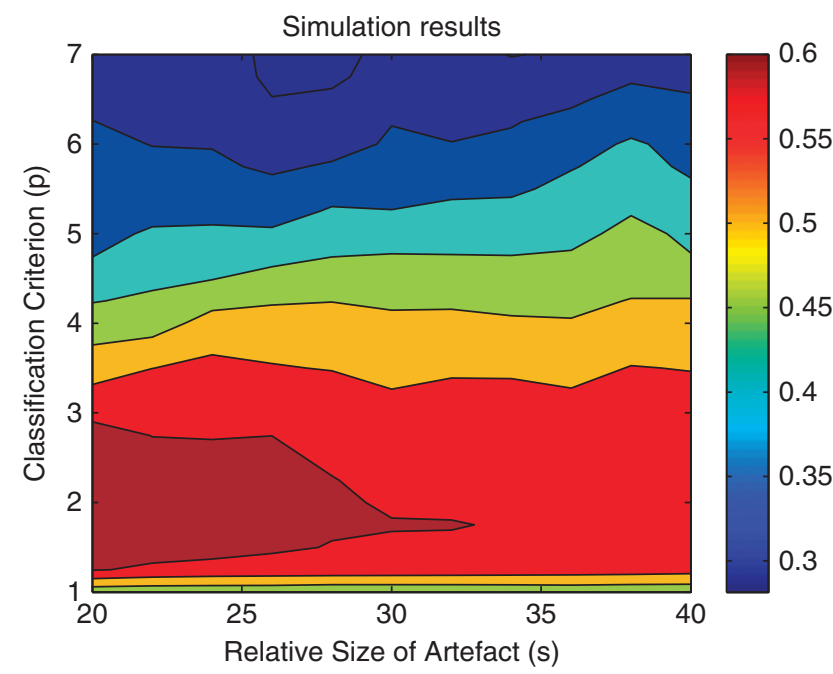

Figure 2. Results of the simulation study. The correlation between the cleaned and original signal without artifact is coded in color for different values of the relative size of the artifact $(s)$ and of the classification criterion $(p)$. midline electrodes, $\mathrm{Fpz}, \mathrm{Fz}, \mathrm{Cz}, \mathrm{Pz}$, and $\mathrm{Oz}$. In the top panel, the original data show an eyeblink artifact occurring between approximately 2 and $4 \mathrm{~s}$ with a maximal impact on the frontopolar electrode. The reconstructed data are shown in the bottom panel of Figure 3. Although traditional ICA-cleaned (red lines) and the proposed ICA+EMD-cleaned data (blue lines) are largely similar, differences do appear on close inspection (see figure insets), and these differences are more pronounced on anterior than on posterior electrodes. When the cleaned frontopolar signals are overlapped with the original data (inset A), it is evident that the combined ICA + EMD method retains more information in the data, especially on shorter time scales. The superiority of the ICA+EMD method over traditional ICA gets more apparent during the time period containing the blink: The ICA-cleaned data show spiky behavior locally that does not seem to be present in the original data, and such spurious peaks are not present in the ICA+EMD-cleaned data (inset B).

To further quantify the improvements of the ICA+EMD method over the traditional ICA method, the correlation coefficient (and the mutual information) between the ICA+EMD-corrected data and the original data was compared with the correlation coefficient between the ICA-only corrected data and the original data. These comparisons focused on the data segments outside the blink intervals, because these segments allow for an assessment of the amount of EEG signal lost by correcting for blinks with either the traditional ICA or the combined ICA+EMD method. For electrode Fpz, where most of the data loss is to be expected, the correlation between the corrected and original data was .81 for the traditional ICA method and .86 for the proposed EMD+ICA method. So, although the amount of data lost with traditional ICA does not seem to be very large, the ICA+EMD method manages to reduce this loss considerably. The mutual information values were .32 and .40 nats between the traditional ICA method and the original data, and between the ICA+EMD method and the original data, respectively. Altogether, these values indicate a considerable increase in the information retained by using the combined ICA+EMD method as compared to the traditional ICA method.

\section{Discussion and Conclusion}

In this article, a novel way to correct for blink-related ocular artifacts has been proposed, based on the combination of EMD and ICA decompositions of multichannel EEG recordings. Whereas ICA provides a spatial filter that conveniently captures widespread blink activity in a small set of ICs, the EMD method can be used as a frequency filter (Flandrin, Rilling, \& Goncalves, 2004) to selectively exclude the artifacts from these ICs and retain useful EEG data that might be present in these ICs as well.

The approach described here is conceptually quite similar to that recently proposed by Castellanos and Makarov (2006), who applied a digital wavelet transform (DWT) to the ICs to separate the blink artifacts from the EEG data. However, on purely theoretical grounds, it might be argued that the EMD is a more suitable decomposition method for EEG/EOG data than the DWT. In essence, EMD is an adaptive method, that is, it does not assume that the same basis for decomposition is suitable for an entire data set, but takes its basis from local dynamics in the data. This property makes it highly suitable for the analysis of nonstationary and nonlinear signals such as EEGs and transient eyeblink artifacts. In contrast, the DWT assumes that the same 
Original Data

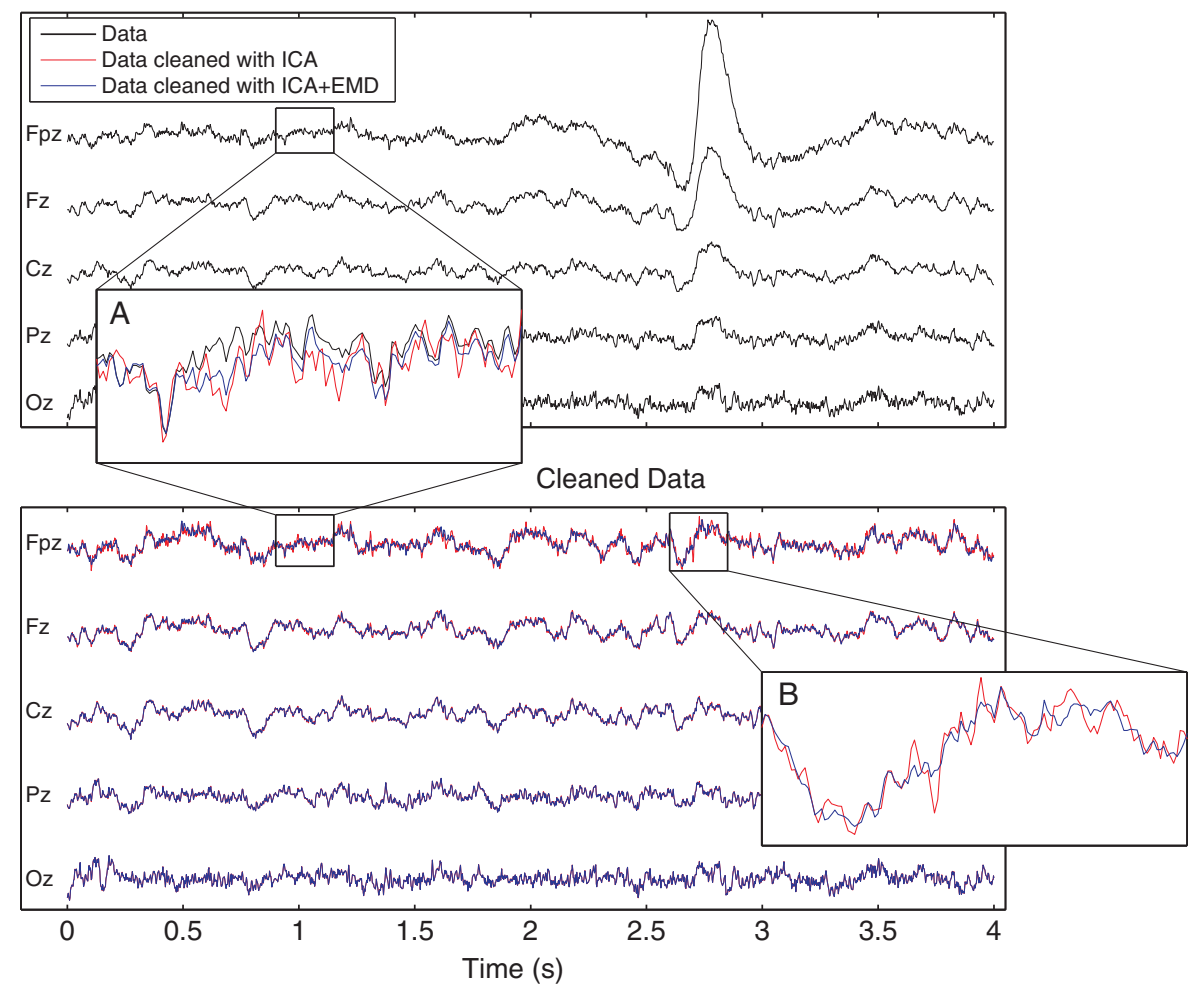

Figure 3. Comparison of ICA and ICA+EMD method for correction of blink artifacts. The top panel shows the midline electrodes from a 64-channel EEG recording containing a blink artifact. The bottom panel shows the same electrodes after removing the independent component (IC) that contained this blink artifact (red line) and after the cleaning this IC using EMD (blue line). Inset A shows in detail the difference between the original signal and the two methods of correcting the blink artifact outside the blink interval. Inset B shows in detail the difference between the two methods of correcting during the blink interval; note the spurious peaks in the ICA cleaned data that do not seem to be present in the original data.

basis is suitable for an entire data set and might yield spurious harmonics because of the nonstationary and nonlinear nature of the signals. Furthermore, performing EMD is computationally straightforward, and the only assumption on the input is that it has at least one minimum and one maximum. Surprisingly, despite the appropriateness of EMD as a suitable technique for EEG analysis, only a few applications have been reported so far (Liang et al., 2005; Sweeney-Reed \& Nasuto, 2007).

Here, it is shown how the EMD method, in combination with ICA, could be applied to correct blink artifacts in EEG data by excluding selective modes in data that contain relatively pure blink activity. Interestingly, the reverse process of excluding the modes that contain EEG data might be of potential interest for another popular method of blink artifact correction based on regression in the time domain (e.g., Gratton et al., 1983). The regression method uses EOG recordings to subtract from each scalp electrode a portion of the EOG signal, but is also likely to subtract some EEG signal, as the EOG recording is most likely not a "pure" recording of blink artifacts. So, EMD has the potential to be applied to EOG signals for selectively eliminating those IMFs associated with EEG, therefore "cleaning" the EOG recordings before the subtraction process and improving the performance of the regression method.

The main limitation of the combined ICA + EMD method is that in addition to eliminating blink-related artifacts in the EEG, it will also eliminate very slow time scale EEG activity, comparable to or slower than the time scale of blink artifacts. ${ }^{1}$ For example, the inset of Figure 1 shows that although the profile of the original signal on a smaller time scale is maintained, the largescale low-frequency trend in the original signal is removed together with the blink-related activity. Potentially, this might greatly reduce EEG activity in the delta (up to $4 \mathrm{~Hz}$ ) range as well as ERP components with a time course of several hundreds of milliseconds. But it must be noted that the loss of slow drift due to the removal of lower order IMFs will occur only if there is a significant overlap between both the frequency characteristics and the spatial distribution of the eyeblink activity and the original EEG as captured by the selected ICs. Importantly, this data loss is relative to the original, uncorrected data; when only ICA is used to correct for blink artifacts, the entire ICs containing blinks would be removed regardless of their frequency content.

Eyeblink artifacts are typically asymmetric with respect to the horizontal axis, that is, the positive peak amplitude is higher than the negative peak amplitude. In contrast, the IMFs are, by definition, symmetrical, as the mean of the upper and lower envelopes is required to be zero at any time point. Therefore, the blink artifact cannot be completely captured by one single IMF but will be distributed over multiple lower order IMFs; see, for example, the bottom panel of Figure 1. For this reason, in the proposed classification criterion, the first IMF that has a $S D$ larger than $p$ times the $S D$ of the first IMF is excluded together with all lower order IMFs, regardless of the $S D$ of these IMFs.

${ }^{1}$ It is shown that for a structured broadband Gaussian process, EMD works as a dyadic filter bank; the mean oscillation of a given IMF is roughly twice that of the previous one (Flandrin et al., 2004). 
In addition to eyeblinks, saccades are another prominent source of artifacts in the EEG. Whereas eyeblinks have a characteristic frequency profile that falls outside the frequency range of interest in the study of many EEG components, the frequencies involved in saccade artifacts typically overlap with this range. For this reason, a frequency-based method for artifact correction is less suitable for the correction of artifacts caused by saccades. Furthermore, the amplitude of saccade artifacts falls generally in the same range as other fluctuations in the raw EEG, rendering a classification method based on the relative size of the $S D$ s of the IMFs problematic.

Finally, eyeblink artifacts are not only a problem in the recording and analysis of EEG data but provide similar problems for the analysis of magnetoencephalogram (MEG) data. Despite some general differences between EEG and MEG signals, the characteristic properties of blink artifacts that the proposed EMD-assisted ICA procedure exploits, that is, its relatively slow time scale and relatively large amplitude com- pared to neurally generated signals, are present in both methods of recording. So, the proposed method for blink artifact correction has potential merit for use in both EEG and MEG data.

In conclusion, combining ICA with EMD provides a useful method to retain EEG activity in ICs representing eyeblink activity. The EMD procedure is an intuitive and computationally straightforward method that separates a signal in modes with different characteristic time scales, allowing for the selective removal of the modes with a slow time scale and large amplitude activity typical of eyeblink artifacts. The instantaneous and adaptive nature of the EMD makes it especially suitable for the analysis of nonstationary and nonlinear processes like EEGs and transient eyeblink artifacts. In this article the effectiveness of the ICA + EMD method was illustrated with both real and simulated data, and a criterion was provided for the classification of IMFs as either containing EEG or blink activity that allows for a fully automated correction of blink artifacts.

\section{REFERENCES}

Barbati, G., Porcaro, C., Zappasodi, F., Rossini, P. M., \& Tecchio, F. (2004). Optimization of an independent component analysis approach for artifact identification and removal in magnetoencephalographic signals. Clinical Neurophysiology, 115, 1220-1232.

Berg, P., \& Scherg, M. (1991). Dipole models of eye movements and blinks. Electroencephalography and Clinical Neurophysiology, 79, 3644.

Castellanos, N. P., \& Makarov, V. A. (2006). Recovering EEG brain signals: Artifact suppression with wavelet enhanced independent component analysis. Journal of Neuroscience Methods, 158, 300-312.

Croft, R. J., \& Barry, R. J. (2000). Removal of ocular artifact from the EEG: A review. Neurophysiologie Clinique, 30, 5-19.

Croft, R. J., Chandler, J. S., Barry, R. J., Cooper, N. R., \& Clarke, A. R. (2005). EOG correction: A comparison of four methods. Psychophysiology, 42, 16-24.

Delorme, A., \& Makeig, S. (2004). EEGLAB: An open source toolbox for analysis of single-trial EEG dynamics including independent component analysis. Journal of Neuroscience Methods, 134, 9-21.

Flandrin, P., Rilling, G., \& Goncalves, P. (2004). Empirical mode decomposition as a filter bank. Signal Processing Letters, IEEE, 11, $112-114$.

Frank, R. M., \& Frishkoff, G. A. (2007). Automated protocol for evaluation of electromagnetic component separation (APECS): Application of a framework for evaluating statistical methods of blink extraction from multichannel EEG. Neurophysiologie Clinique, 118, $80-97$.

Gratton, G., Coles, M. G., \& Donchin, E. (1983). A new method for offline removal of ocular artifact. Electroencephalography and Clinical Neurophysiology, 55, 468-484.

Hoffmann, S., \& Falkenstein, M. (2008). The correction of eye blink artefacts in the EEG: A comparison of two prominent methods. PLoS ONE, 3, e3004.

Huang, N. E., Shen, Z., Long, S. R., Wu, M. C., Shih, H. H., Zheng, Q., et al. (1998). The empirical mode decomposition and the Hilbert spectrum for nonlinear and non-stationary time series analysis. Proceedings of the Royal Society A: Mathematical, Physical and Engineering Sciences, 454, 903-995.

Ille, N., Berg, P., \& Scherg, M. (2002). Artifact correction of the ongoing EEG using spatial filters based on artifact and brain signal topographies. Journal of Clinical Neurophysiology, 19, 113-24.
Joyce, C. A., Gorodnitsky, I. F., \& Kutas, M. (2004). Automatic removal of eye movement and blink artifacts from EEG data using blind component separation. Psychophysiology, 41, 313-325.

Jung, T. P., Makeig, S., Humphries, C., Lee, T. W., McKeown, M. J., Iragui, V., et al. (2000). Removing electroencephalographic artifacts by blind source separation. Psychophysiology, 37, 163-178.

$\mathrm{Li}, \mathrm{W}$. (1990). Mutual information functions versus correlation functions. Journal of Statistical Physics, 60, 823-837.

Liang, H., Bressler, S. L., Buffalo, E. A., Desimone, R., \& Fries, P. (2005). Empirical mode decomposition of field potentials from macaque V4 in visual spatial attention. Biological Cybernetics, 92, 380392.

Lindsen, J. P., Jones, R., Shimojo, S., \& Bhattacharya, J. (2010). Neural components underlying subjective preferential decision making. $\mathrm{Ne}$ uroImage, 50, 1626-1632.

Matsuo, F., Peters, J. F., \& Reilly, E. L. (1975). Electrical phenomena associated with movements of the eyelid. Electroencephalography and Clinical Neurophysiology, 38, 507-511.

Picton, T. W., van Roon, P., Armilio, M. L., Berg, P., Ille, N., \& Scherg, M. (2000). The correction of ocular artifacts: A topographic perspective. Clinical Neurophysiology, 111, 53-65.

Quilter, P. M., MacGillivray, B. B., \& Wadbrook, D. G. (1977). The removal of eye movement artifact from the EEG signals using correlation techniques. Random Signal Analysis, IEEE Conference Publication, 159, 93-100.

Schlogl, A., Keinrath, C., Zimmermann, D., Scherer, R., Leeb, R., \& Pfurtscheller, G. (2007). A fully automated correction method of EOG artifacts in EEG recordings. Clinical Neurophysiology, 118, 98 104.

Sweeney-Reed, C. M., \& Nasuto, S. J. (2007). A novel approach to the detection of synchronisation in EEG based on empirical mode decomposition. Journal of Computational Neuroscience, 23, 79-111.

Verleger, R., Gasser, T., \& Mocks, J. (1982). Correction of EOG artifacts in event-related potentials of the EEG: Aspects of reliability and validity. Psychophysiology, 19, 472-480.

Vigario, R. N. (1997). Extraction of ocular artifacts from EEG using independent component analysis. Electroencephalography and Clinical Neurophysiology, 103, 395-404.

(Received February 26, 2009; ACCePted September 30, 2009) 